\title{
Uma Proposta para a Porta Toffoli usando QND para Qubits baseados em Polarização e suas Aplicações
}

\author{
Samy C. Policarpo ${ }^{1}$, João Batista R. Silva ${ }^{1}$ \\ ${ }^{1}$ Depart. de Engenharia de Teleinformática - Universidade Federal do Ceará (UFC) \\ Caixa Postal - 60.440 - 900 - Fortaleza - CE - Brazil \\ samycleverp@gmail.com, joaobrs@ufc.br
}

\begin{abstract}
An optical apparatus to implement the Toffoli gate is presented for polarization-based qubits using quantum non-demolition measurement (QND) with a probability of success of $0.95(19 / 20)$ using real optical devices. From this apparatus it is possible to implement universal gates for reversible classical and quantum computing.
\end{abstract}

Resumo. Um aparato óptico para implementar a porta Toffoli é apresentado para qubits baseados em polarização usando medição quântica não demolidora (QND) com uma probabilidade de sucesso de 0,95 (19/20) usando dispositivos ópticos reais. A partir deste aparato é possível implementar portas universais para computação quântica e clássica reversível.

\section{Introdução}

A versatilidade da porta Toffoli [Toffoli 1980] torna-a uma porta quântica de múltiplos qubits muito desejável em sua implementação para computação quântica em larga escala [Nielsen 2002], [ Gottesman 1998]. Quando associada a portas de um qubit, a porta Toffoli é universal para processamento de informações quânticas. Infelizmente, implementar uma porta Toffoli de alta fidelidade é difícil, pois envolve interação não linear de três qubits. A implementação da porta Toffoli com apenas portas de um qubit e de dois qubits requer seis portas NOT controladas (CNOT) e dez operações de um qubit [Barenco 1995]. Apesar da dificuldade de implementação, a porta Toffoli foi implementada com: ressonância magnética nuclear [Cory 1998], óptica linear [Lanyon 2009], sistemas de armadilha de íons [Monz 2009] e circuitos supercondutores [Mariantoni 2011], [Fedorov 2012].

Portanto, neste trabalho apresentamos uma nova proposta para implementar a porta Toffoli para qubits de polarização usando medição quântica não demolidora (QND) baseado em [Lin 2009]. Este trabalho está dividido nas seguintes seções: a Seção 2 apresenta um sistema óptica capaz de implementar a porta Toffoli para qubits baseados em polarização usando QND, é analisada seu desempenho com uso de dispositivos ópticos reais e que é possível usá-lo para implementar outras operações lógicas reversíveis; e na Seção 3 são apresentadas as conclusões.

\section{Sistema óptica da porta Toffoli}

A porta Toffoli (porta $\mathrm{C}^{2}$-NOT) é uma porta reversível de 3 qubits que realiza a

O presente trabalho foi realizado com apoio da Coordenação de Aperfeiçoamento de Pessoal de Nível Superior - Brasil (CAPES) Código de Financiamento 001 e pelo INCT-IQ. 
operação NOT no terceiro qubit somente quando os dois primeiros qubits são $|1\rangle \mathrm{e}$, caso contrário, nenhuma inalteração é feita. A representação do circuito da porta $\mathrm{C}^{2}-\mathrm{NOT}$, sua tabela verdade e matriz são mostradas na Figura 1, respectivamente. As portas Toffoli e Hadamard são universais para computação quântica [Aharonov 2003].

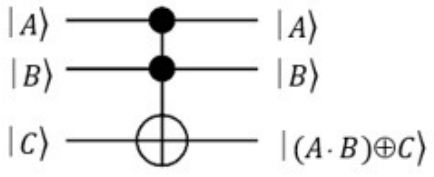

(a)

\begin{tabular}{|c|c|c|c|c|c|}
\hline \multicolumn{3}{|c|}{ Input } & \multicolumn{3}{c|}{ Output } \\
\hline $\boldsymbol{A}$ & $\boldsymbol{B}$ & $\boldsymbol{C}$ & $\boldsymbol{A}$ & $\boldsymbol{B}$ & $(\boldsymbol{A} \cdot \boldsymbol{B}) \oplus \boldsymbol{C}$ \\
\hline 0 & 0 & 0 & 0 & 0 & 0 \\
\hline 0 & 0 & 1 & 0 & 0 & 1 \\
\hline 0 & 1 & 0 & 0 & 1 & 0 \\
\hline 0 & 1 & 1 & 0 & 1 & 1 \\
\hline 1 & 0 & 0 & 1 & 0 & 0 \\
\hline 1 & 0 & 1 & 1 & 0 & 1 \\
\hline 1 & 1 & 0 & 1 & 1 & 1 \\
\hline 1 & 1 & 1 & 1 & 1 & 0 \\
\hline
\end{tabular}

(b)

$\left[\begin{array}{llllllll}1 & 0 & 0 & 0 & 0 & 0 & 0 & 0 \\ 0 & 1 & 0 & 0 & 0 & 0 & 0 & 0 \\ 0 & 0 & 1 & 0 & 0 & 0 & 0 & 0 \\ 0 & 0 & 0 & 1 & 0 & 0 & 0 & 0 \\ 0 & 0 & 0 & 0 & 1 & 0 & 0 & 0 \\ 0 & 0 & 0 & 0 & 0 & 1 & 0 & 0 \\ 0 & 0 & 0 & 0 & 0 & 0 & 0 & 1 \\ 0 & 0 & 0 & 0 & 0 & 0 & 1 & 0\end{array}\right]$

(c)

Figura 1. (a) circuito, (b) tabela verdade e (c) forma matricial que representa uma porta Toffoli.

Um elemento essencial usado neste trabalho para implementar a porta Toffoli é uma medição quântica não demolidora (QND) que pode introduzir uma fase $\theta$ ao estado coerente $|\alpha\rangle$ quando este e um único fóton $|1\rangle$ propagam em um meio com alto nãolinearidade cruzada de Kerr, conforme mostrado na Figura 2. O estado coerente $|\alpha\rangle$ (teste) sobre uma mudança de fase que depende do tempo de interação $t$ e da força do coeficiente não linear:

$$
e^{i t H_{Q N D} / \hbar}|1\rangle_{s}|\alpha\rangle_{p}=|1\rangle_{s}\left|\alpha e^{i \kappa t}\right\rangle_{p} .
$$

Em (1) $H_{Q N D}=\hbar \kappa a_{s}^{\dagger} a_{s} a_{p}^{\dagger} a_{p}$ é o Hamiltoniano, $\kappa$ é a força da não linearidade, $a_{s}^{\dagger}\left(a_{s}\right)$ são os operadores de criação (aniquilação) do modo de sinal, enquanto $a_{p}^{\dagger}\left(a_{p}\right)$ é o operador de criação (aniquilação) do modo de teste. A condição $\theta=k t>\pi /\left(2\langle n\rangle^{1 / 2}\right)$ deve ser satisfeita [Grangier 1998], [Kok 2002], onde $\langle n\rangle=|\alpha|^{2}$ é o número médio de fótons do estado coerente $|\alpha\rangle$. Portanto, a amplitude do estado coerente $\alpha$ deve ser grande o suficiente para obedecer à condição $|\alpha| \kappa t\rangle 1$.

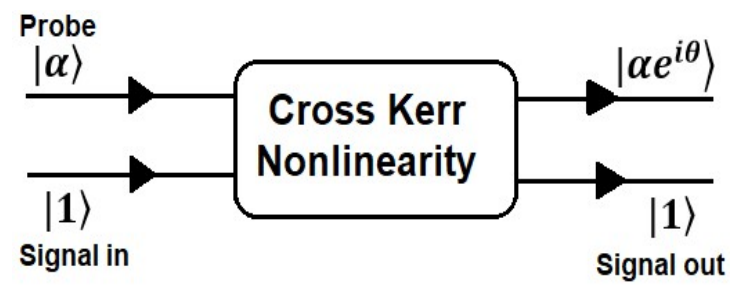

Figura 2. 0 estado coerente sofre um deslocamento de fase $\theta$ devido à copropagação com o fóton único em um meio com alta não linearidade cruzada de Kerr.

A configuração óptica básica capaz de implementar a porta Toffoli para qubit baseado em polarização usando medição quântica não demolidora (QND) é mostrada na Figura 3. Este sistema tem: dois divisores de feixe balanceados (BS), quatro divisores de feixe de polarização $(\mathrm{PBS})$, dois rotadores de polarização $(R)$, dois fotodetectores $\left(\mathrm{D}_{1}\right.$ 
e $\left.\mathrm{D}_{2}\right)$, dois meios do tipo mostrado na Figura $2\left(\theta_{A}\right.$ e $\left.\theta_{B}\right)$ e um circuito de decisão (DC). $\mathrm{O}$ subsistema $R(\pi / 2)-\mathrm{PBS}_{2}-\mathrm{D}_{1} / \mathrm{D}_{2}$ funciona com um polarímetro que mede $\mathrm{o}$ parâmetro de Stokes $S_{1}$ do estado bimodal coerente $|\alpha, \beta\rangle_{H V}$ onde

$$
\left\langle\hat{S}_{1}\right\rangle=|\alpha|^{2}-|\beta|^{2} \text {. }
$$

Dependendo do resultado medido por este polarímetro, o DC ligará ou não $R_{1}(\pi / 2)$ que funciona como uma porta NOT no qubit $|C\rangle$ baseado em polarização. As informações lógicas ' 0 ' e '1' são codificadas nos estados de polarização ortogonal ( $H$-horizontal e $V$ vertical) para qubit único, ou seja, o bit '0' é representado por $|H\rangle(|H\rangle=|0\rangle)$ e o bit '1' para $|V\rangle(|V\rangle=|1\rangle)$.

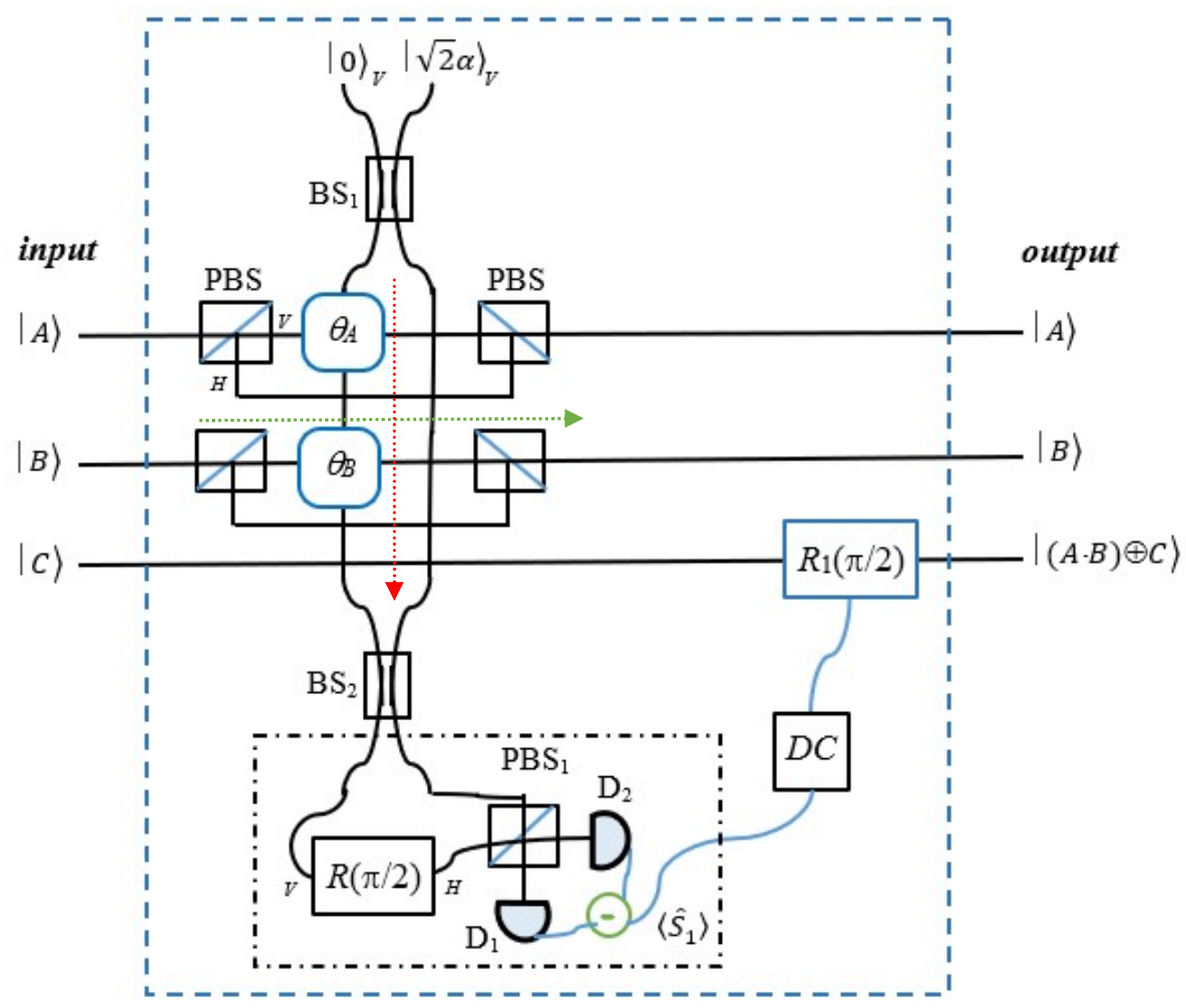

Figura 3. O esquema óptico baseado em fibra que é possível implementar a porta Toffoli para qubit baseado em polarização usando medição quântica não demolidora.

Conforme mostrado na Figura 3, os estados de entrada $|A\rangle$ e $|B\rangle$ são os qubits de controle e o estado $|C\rangle$ é o qubit alvo da porta Toffoli, todos se propagam da esquerda para direita (sentido da seta na cor verde). O estado coerente bimodal, que se propaga de cima para baixo (sentido da seta na cor vermelha), antes do $\mathrm{BS}_{2}$ é $\left|-\alpha e^{j \Sigma \theta}, \alpha\right\rangle_{V V}$, após a interação com $|A\rangle(|B\rangle)$ que se propagou através do caminho PBS - $\theta_{A}\left(\theta_{B}\right)$ - PBS, e após sofrer interferência em $\mathrm{BS}_{2}$ e antes de $\mathrm{PBS}_{1}$ ser

$\left|\alpha\left(\frac{e^{j \Sigma \theta}+1}{\sqrt{2}}\right), \alpha\left(\frac{e^{j \Sigma \theta}-1}{\sqrt{2}}\right)\right\rangle_{H V}$, 
onde $\Sigma \theta=\theta_{A}+\theta_{B}$ se $|A\rangle=|V\rangle\left(\theta_{A} \neq 0\right)$ e $|B\rangle=|V\rangle\left(\theta_{B} \neq 0\right)$ ou $\Sigma \theta=\theta_{A}\left(\Sigma \theta=\theta_{B}\right)$ se $|A\rangle$ $=|V\rangle$ e $|B\rangle=|H\rangle(|A\rangle=|H\rangle$ e $|B\rangle=|V\rangle)$, caso contrário, $\Sigma \theta=0$ se $|A\rangle=|H\rangle$ e $|B\rangle=|H\rangle$. Aplicando (3) em (2), o valor médio medido de $\left\langle\hat{S}_{1}\right\rangle$ é

$$
S_{1}=\left\langle\hat{S}_{1}\right\rangle=2|\alpha|^{2} \cos (\Sigma \theta) \text {. }
$$

Em (4) podemos observar que se $\theta_{A}=\theta_{B}=\pi / 2(\Sigma \theta=\pi)$ implica que $S_{1}<0$, ou seja, ambos os qubits de controle, $|A\rangle$ e $|B\rangle$ são iguais a $|V\rangle$, então o circuito de decisão DC, conforme mostrado na Figura 3, ativa o rotacionador de polarização $R_{1}$ (porta NOT) para alterar o estado de polarização do qubit alvo $|C\rangle$. Se $S_{1} \geq 0, R_{1}$ permanece desligado. Assim, o circuito mostrado na Figura 3 é capaz de implementar a porta Toffoli para qubits baseados em polarização.

Se considerarmos que apenas dispositivos Kerr cruzado são dispositivos reais e podem introduzir uma variação global de fase $\delta$, então podemos calcular a probabilidade de sucesso do sistema proposto na Figura 3. De (3), o estado na saída na Figura 3, antes da medição $S_{1}$, será $\left|\Psi_{A B \delta}\right\rangle=|A B\rangle\left|\alpha\left(e^{j(\Sigma \theta \pm \delta)}+1\right) / \sqrt{ } 2\right\rangle\left|\alpha\left(e^{j(\Sigma \theta \pm \delta)}-1\right) / \sqrt{ } 2\right\rangle_{H V}$. Então a probabilidade de sucesso $P_{S}$ será dada por:

$$
P_{s}=\frac{1}{4}\left(\left\langle\Psi_{H H 0}\left|\rho_{H H \delta}\right| \Psi_{H H 0}\right\rangle+\left\langle\Psi_{H V 0}\left|\rho_{H V \delta}\right| \Psi_{H V 0}\right\rangle+\left\langle\Psi_{V H 0}\left|\rho_{V H \delta}\right| \Psi_{V H 0}\right\rangle+\left\langle\Psi_{V V 0}\left|\rho_{V V \delta}\right| \Psi_{V V 0}\right\rangle\right),
$$

onde $\rho_{A B \delta}=\left|\Psi_{A B \delta}\right\rangle\left\langle\Psi_{A B \delta}\right|$ é a matriz de densidade de $\left|\Psi_{A B \delta}\right\rangle$ para $\delta \neq 0$. Assim, para fotodetectores com uma probabilidade de escuro $p_{d}$ e eficiência quântica $\eta$, de (5) temos

$$
P_{s}=\frac{\left(1-p_{d}\right)^{2}}{2} e^{-4|\alpha|^{2} \sin ^{2}(\delta / 2)}\left[\left(1-e^{-2|\alpha|^{2} \eta \cos ^{2}(\delta / 2)}\right) e^{-2|\alpha|^{2} \eta \sin ^{2}(\delta / 2)}+\left(1-e^{-2|\alpha|^{2} \eta[1+\sin (\delta)]}\right)\left(1-e^{-2|\alpha|^{2} \eta[1-\sin (\delta)]}\right)\right]
$$

Em (6) podemos inferir que o nosso esquema pode ter uma probabilidade de sucesso de $19 / 20$ (para $\delta=0,04$ radianos com $|\alpha|^{2}=25, p_{d}=10^{-6}$ e $\eta=0,9$ ) em comparação ao esquema proposto por Lin et al. [Lin 2009] que apresentou uma probabilidade de sucesso de $2 / 23$.

A porta Toffoli mostrada na Figura 3 pode ser usada para simular portas lógicas clássicas irreversíveis, por exemplo: se $|C\rangle=|H\rangle$, o sistema implementa a função AND reversível para dois qubits; se $|C\rangle=|V\rangle$, o sistema implementa a função NAND reversível; e se $|A\rangle=|V\rangle$ e $|C\rangle=|H\rangle$, temos uma FANOUT. Com essas duas últimas operações, é possível simular todos os outros elementos em um circuito clássico e, portanto, um circuito clássico arbitrário pode ser simulado por um circuito reversível equivalente [Nielsen 2002].

\section{Conclusão}

Este trabalho apresentou um sistema óptico usando QND que implementa uma porta Toffoli para qubits baseados em polarização com um desempenho de $95 \%$ para dispositivos ópticos ativos reais e $|\alpha|^{2}=25$. Além das portas descritas (AND e NAND reversíveis) que são possíveis de implementar com o sistema proposto, também é possível usá-lo para simular a porta FANOUT que combinada com a NAND são portas universais para computação reversível. 


\section{Referências}

Aharonov, Dorit. "A Simple Proof that Toffoli and Hadamard are Quantum Universal”, arXiv:quant-ph/0301040, (2003-01-09).

Barenco, A. et al. "Elementary gates for quantum computation", Phys. Rev. A 52, 3457-3467 (1995).

Cory, D. G. et al. "Experimental quantum error correction”, Phys. Rev. Lett. 81, 21522155 (1998).

Fedorov, A., Steffen, L., Baur, M. et al. "Implementation of a Toffoli gate with superconducting circuits", Nature 481, 170-172 (2012).

Gottesman D., "Theory of fault-tolerant quantum computation", Phys. Rev. A 57, 127, (1998).

Lanyon, B. P. et al. "Simplifying quantum logic using higher-dimensional Hilbert spaces", Nature Phys. 5, 134-140 (2009).

Nielsen, M. A. and Chuang, I. L., "Quantum Computation and Quantum Information", Cambridge University Press, Cambridge, U.K., 2002.

Mariantoni, M., et al, "Implementing the quantum von Neumann architecture with superconducting circuits", Science 334, 61-65 (2011).

Monz, T. et al, "Realization of the quantum Toffoli gate with trapped ions", Phys. Rev. Lett. 102, 040501 (2009).

Grangier, P., Levenson, J. A. and Poizat, JP., "Quantum non-demolition measurements in optics", Nature, 396, 537, 1998.

Kok, P., Lee, H. and Dowling, J. P., "Single-photon quantum-nondemolition detectors constructed with linear optics and projective measurements", Phys. Rev. A, 66, 063814, 2002.

Toffoli T., "Reversible Computing Automata", Languages and Programming (Lecture Notes in Computer Science vol 85) ed. J. W. de Bakker and J. van Leeuwen (Berlin: Springer), 1980, pp 632-44.

Lin, Q. and Li, J., "Quantum control gates with weak cross-Kerr nonlinearity", Phys. Rev. A 79, 022301 - Published 2 February 2009. 